forward direction expressed in roentgens per min. and milliampere at one meter from the target and $I_{\theta}$, the intensity at angle $\theta$ relative to that in the forward direction, and if we recognize the fact that the coefficient for true absorption in air does not depend appreciably on angle, we may write

$$
P_{1}=1.59 \times 10^{-5}\left(I_{f} / \mu\right) \int_{0}^{\pi} I_{\theta} \sin \theta d \theta \mathrm{watt} / \mathrm{ma} \text {. }
$$

Then the efficiency of $\mathrm{x}$-ray production is

$$
\eta=1.59\left(I_{f} / \mu V\right) \int_{0}^{\pi} I_{\theta} \sin \theta d \theta \text { percent }
$$

where $V$ is the tube drop in volts.

Experimental data already presented make it possible to calculate the efficiency at three voltages: $2.35,1.63$, and $0.90 \mathrm{Mv}$. Values of $I_{f}$ are obtained from Fig. 10 and values of the integral are calculated from the dashed curves of Fig. 7. The values of $\mu$, the linear coefficient for true absorption in air of the heterogeneous radiation incident upon the thimble chamber, were calculated as weighted averages from the corresponding coefficients for homogeneous radiation. From curves similar to those shown in Fig. 6 it is possible to obtain directly factors to correct for true absorption of $x$-ray power in the target. The ratio of the area under the Kramers' distribution to that under the reduced distribution gives the factor by which the power has been reduced in passage through the target. The correction is not serious except at the lowest voltage. Values of $I_{f}$, the integral, $\mu$, the correction factor, and $\eta$ are presented in Table III.

The theoretical values for the efficiency given in the last column were computed from curves calculated from Bethe and Heitler's theory by Arcimovic and Chramov. ${ }^{20}$ In view of limitations in the theory when applied to targets of high atomic number, the agreement between theory and experiment throughout this voltage range may be considered quite satisfactory.

The writers are indebted to Dr. G. G. Harvey for helpful discussions and to Mr. Zigmond Wilchinsky, Mr. W. B. Nowak, and Mr. Howard Rowland for much capable assistance in making measurements and calculations. The research was supported in part by grants from Research Corporation and the Carnegie Corporation of New York.

${ }^{20}$ L. A. Arcimovic and V. A. Chramov, Comptes Rendus Acad. Sci. U.S.S.R., 18, 415 (1938).

\title{
Errata: Quantization of Molecules, Inter- and Intramolecular Forces
}

\author{
[Phys. Rev. 63, 309 (1943)] \\ Kasimir Fajans and Theodore Berlin \\ Department of Chemistry, University of Michigan, Ann Arbor, 'Michigan
}

THE sentence on page 310, left column, lines 8-6 from the bottom should read: "In Fig. 1A, two $\mathrm{Li}^{+}$cores are placed at the internuclear distance $0.74 \mathrm{~A}$ of $\mathrm{H}_{2}(n=\mathrm{I})$."

On page 311 , left column, line 7 from the bottom should have $n=3$ instead of $n=$ III.

\section{Erratum: The Carbon Arc in Oxygen for the Spectrochemical Determination of Potassium}

[Phys. Rev. 63, 322 (1943)]

\author{
L. T. STEadman
}

Department of Radiology, The University of Rochester, School of Medicine and Dentistry, Rochester, New York

7 HE last paragraph, beginning line 8 from the bottom, should read: "To the contains 33.0 gamma (micrograms) Na, 2.0 gamma $\mathrm{K}, 1.0$ gamma $\mathrm{Ca}, 0.27$ gamma $\mathrm{Mg}$, and 1.3 gamma $\mathrm{P}$. Also, 2.5 gamma $\mathrm{Rb}$ is added as the internal standard for $\mathrm{K}$ and 100 gamma $\mathrm{Cd}$ as the internal standard for all the other elements. The spectrum lines measured are $\mathrm{Na}$ 2680.3A, K 4044.1A, Ca 3006.9A, Mg 2783.0A, P 2535.6A, Rb 4201.8A, and Cd 2677.6A." 